\title{
Papain-like 2 protease of coronavirus and innate immune response
}

\author{
Young Jin Jeon \\ Department of Pharmacology, Chosun University College of Medicine, Gwangju, Korea
}

Received June 9, 2020

Revised June 17, 2020

Accepted June 19, 2020

Corresponding author

Young Jin Jeon

Department of Pharmacology,

Chosun University College of

Medicine, 309, Pilmun-daero,

Dong-gu, Gwangju 61452, Korea

Tel: $+82-62-230-6338$

Fax: +82-62-232-9213

E-mail: yjjeon@chosun.ac.kr

ORCID:

http://orcid.org/0000-0002-5602-3100

\begin{abstract}
Over the past few decades, certain coronaviruses have become threat to humans. Coronaviruses such as severe acute respiratory syndrome-related coronavirus (SARS-CoV) and Middle East respiratory syndrome-related coronavirus (MERS-CoV) have caused lethal outbreaks in 2003 and 2012, respectively. In 2019, severe infectious coronavirus outbreak with unusual pneumonia was reported in Wuhan, China, which later became pandemic. Due to the similarity of coronavirus disease 2019 (COVID-19) to SARS-CoV, the virus was termed as SARS-CoV-2. Although viral proteases such as papain-like 2 protease $\left(P L 2^{\text {Pro }}\right.$ ) are essential for virulence of SARS-CoV, limited information is available on the role of protease in viral pathogenesis. This article highlights the significance of ubiquitination in signal transduction for innate immune responses and the importance of deubiquitinase activity of PL2 ${ }^{\text {Pro }}$ in inhibiting host antiviral activities.
\end{abstract}

Keywords: COVID-19; Papain-like 2 protease; SARS-CoV-2; Deubiquitinase

\section{INTRODUCTION}

Coronavirus disease 2019 (COVID-19), which was termed by World Health Organization (WHO), originated in Wuhan City in the Hubei Province of China. COVID-19 has recently been identified to be caused by human coronavirus, which belongs to genera Betacoronavirus. Due to its similarity to severe acute respiratory syndrome-related coronavirus (SARS-CoV), the virus causing COVID-19 was termed as SARS-CoV-2. In this review, we provide the genetic and phenotypic features of SARS-CoV-2 and discuss papain-like 2 protease $\left(\mathrm{PL}_{2}{ }^{\mathrm{Pro}}\right)$ and host immune response.

\section{COMPARISON OF SARS-COV-2, SARS- COV, AND MERS-COV}

Coronavirus is a large and diverse family of single-stranded RNA viruses that are $26-32 \mathrm{~kb}$ in size [1]. Coronaviruses include a broad spectrum of hosts and have been reported to trigger diseases related to upper respiratory tract, gastrointestinal tract, liver, and central nervous system [2].

Coronaviruses have been observed to infect many avian and mammalian species. Moreover, they have been shown to have capability of crossing the species barrier, infecting humans, and causing severe symptoms. The outbreak of SARS in 2003 and Middle East respiratory syndrome (MERS) in 2012 have proved the lethality of coronavirus infection in humans [3]. Studies showed that SARS-CoV and MERS-CoV 
were likely originated from bats (Fig. 1). Before infecting humans, SARS-CoV and MERS-CoV have been suggested to infect civet cat and camel, respectively. The Chinese Center for Disease Control and Prevention reported a new zoonotic human coronavirus on $9^{\text {th }}$ January 2020 [4]. Although the initial infected cases have been associated with Hunan South China Seafood Market, the source of SARS-CoV-2 is still unknown (Fig. 1). Recent studies have suggested that the intermediate carriers may be pangolins [5]; however, according to the World Health Organization, the original

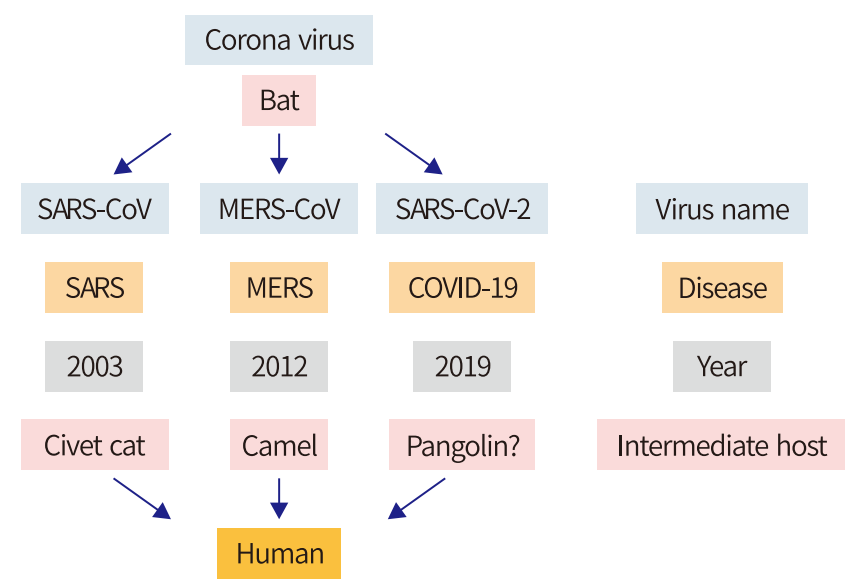

Fig. 1. Illustration of name and transmission of coronaviruses. Coronaviruses are a group of related viruses that cause diseases in birds and mammals. In humans, coronavirus infects respiratory tract and causes mild to lethal illness. Mild symptoms include common cold while severe lethal variations cause SARS, MERS, and COVID-19. The intermediate carriers of SARS and MERS were found to be civet cat and camel, respectively. Recent studies have suggested that the intermediate carrier of COVID-19 may be pangolins. SARS: severe acute respiratory syndrome, MERS: Middle East respiratory syndrome, COVID-19: coronavirus disease-2019. source is still unknown [6].

\section{VIRAL STRUCTURE AND INFECTION}

SARS-CoV-2 is a spherical, enveloped particle containing single-stranded RNA, which is associated with a nucleoprotein. The envelope has been reported to exhibit clubshaped glycoprotein projections called spikes. Coronaviruses have been shown to have large RNA genomes (26-32 kb). The viral genome has been reported to have unique N-terminal fragment, expressing 16 non-structural proteins and C-terminal structural proteins that includes spike, envelop, membrane, and nucleocapsid (Fig. 2). Furthermore, coronavirus genomes have been reported to usually enclose about six ORFs.

The first ORF (ORF1a/b) occupies two-thirds of the whole genome length and encodes 16 non-structural proteins (nsp1-16). The four key structural proteins are encoded by ORFs, 10 and 11, which are on the remaining one-third of the genome near 3 '-end. These mature proteins have been shown to play important roles in genome maintenance and replication of virus $[7,8]$. The most abundant structural protein, membrane glycoprotein, is known to span the membrane bilayer three times, which leaves The N-terminal and C-terminal domain exposed to the outside and inside of the virus, respectively. The membrane protein has been reported to play a significant role in the intracellular formation of viral particles.

The spike protein is a membrane glycoprotein that is

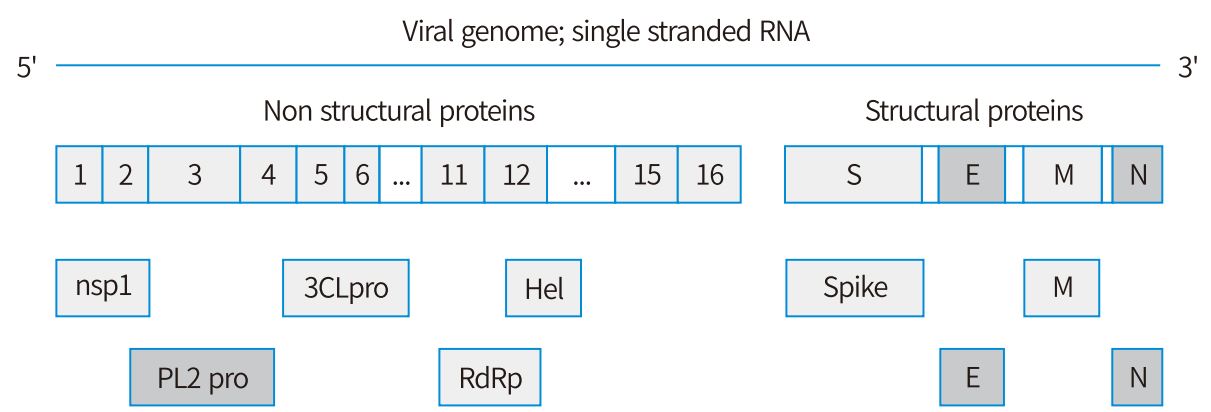

Fig. 2. Viral genome arrangement and gene products. Coronavirus genome comprises $\sim 30 \mathrm{~kb}$ of single-stranded RNA. The $5^{\prime}$ two-third of the coronavirus genome comprises of ORF1a and ORF1b. ORF1a encodes polyprotein 1a (Nsp1-11) while ORF1a and ORF1b together encode polyprotein 1ab (Nsp1-16) through ribosomal frameshift mechanism, which overreads the stop codon of ORF1a. The 3' one-third encodes structural proteins including S, E, M, and $\mathrm{N}$; and accessory proteins. The polyproteins, pp1a and pp1ab are processed by the viral proteases PL1 ${ }^{\text {Pro }}, \mathrm{PL2}^{\text {Pro }}$ (both domains of Nsp3), and $\mathrm{M}^{\text {Pro }}$ (3CL ${ }^{\text {Pro }}$, Nsp5). Nsp1 possibly interacts with cyclophilins. PL2 ${ }^{\text {Pro }}$ functions as a protease, deubiquitinase, and delSGylase. $3 \mathrm{CL}^{\text {Pro }}$ is a cysteine protease and cleaves the polyprotein at two self-cleavage sites. RdRp catalyzes the replication of RNA using RNA template. Hel functions to unpackage genes. S binds to cell receptor. Structural proteins; E, M, and N are important for viral assembly. nsp: nonstructural protein, PL2 ${ }^{\text {Pro: }}$ papain-like 2 protease, $3 \mathrm{CL}^{\text {Pro: }}$ : $3 \mathrm{C}$-like main protease, RdRp: RNA-dependent RNA polymerase, Hel: helicase, S: spike, E: envelop, M: membrane, N: nucleoprotein. 
crucial for penetrating host cells. Spike protein of SARS$\mathrm{CoV}-2$ has been suggested to bind to angiotensin-converting enzyme 2 (ACE2) (Fig. 3). On attachment, the spikes are primed by host enzyme called transmembrane protease, serine 2 (TMPRSS2). This proteolytic activity has been reported to activate spike and allow SARS-CoV-2 to success-

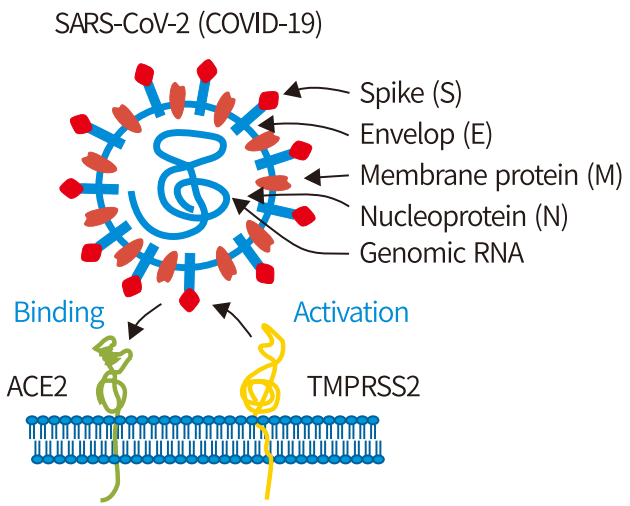

Fig. 3. Structure of coronavirus. SARS-CoV-2 contains glycoproteins (S, E, $\mathrm{M}$, and $\mathrm{N}$ ) and a single-stranded genomic RNA. Both, SARS-CoV-2 and SARS-CoV utilize cellular transmembrane protease, serine 2 (TMPRSS2) for their spike activation and angiotensin-converting enzyme 2 (ACE2) for attachment. SARS-CoV-2: severe acute respiratory syndrome-related coronavirus 2. fully enter and infect cells $[9,10]$.

\section{PAPAIN-LIKE PROTEASE 2 AND INNATE IMMUNE RESPONSE}

Interestingly, PL2 ${ }^{\text {Pro }}$ has three enzyme activities including proteolysis, deubiquitination, and deISGylation. PL2 ${ }^{\text {Pro }} \mathrm{di}^{-}$ gests polypeptide of virus and can produce several proteins. Deubiquitination and deISGylation are vital functions of protease that cause reduction in host immune response. Since ubiquitin (Ub) and interferon-stimulated gene 15 (ISG15) are crucial for innate antiviral immunity, coronaviruses have been shown with tendency to inhibit the conjugation of Ub or ISG15 to its target proteins, and remove Ub or ISG15 from ubiquitinated or ISGylated proteins, respectively [11-13].

Ubiquitination is a chemical process, which involves covalent and reversible addition of a 76-amino acid protein, $\mathrm{Ub}$, to lysine or other residues. Ubiquitination of $\mathrm{Ub}$ itself at its lysine residues $(\mathrm{K} 6 / \mathrm{K} 11 / \mathrm{K} 27 / \mathrm{K} 29 / \mathrm{K} 33 / \mathrm{K} 48 / \mathrm{K} 63)$ or its amino-terminal methionine produces different types of $\mathrm{Ub}$ chains with distinct functions. For instance, K48-linked Ub

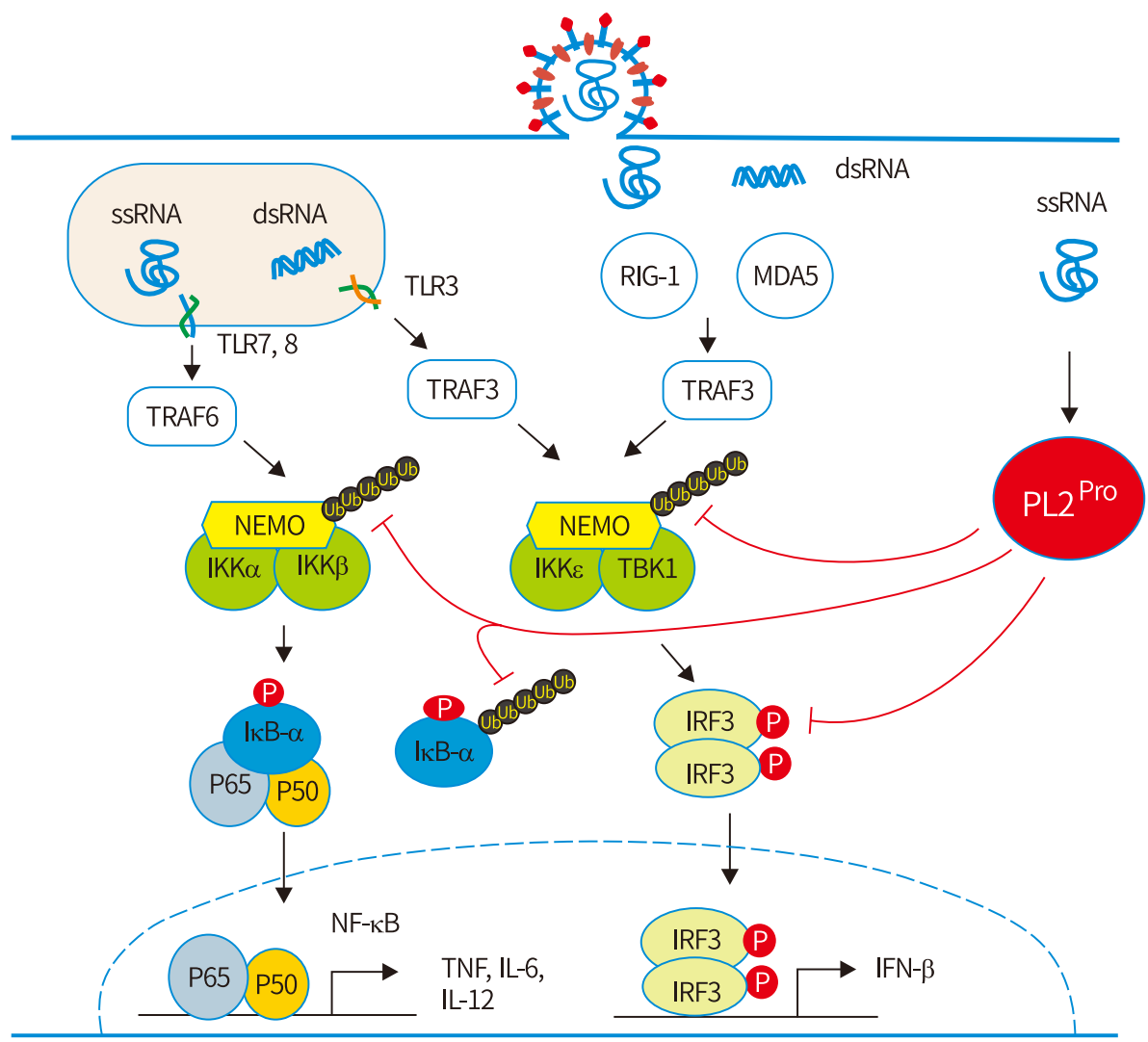

Fig. 4. Antiviral signaling pathways and the potential targets of SARS-CoV PL2 ${ }^{\text {Pro }}$. TLR7, TLR8, TLR3, RIG-1, and MDA5 are activated by endosomal and cytosolic viral RNA species. TLR7 and TLR8 activate TRAF6 while TLR3 activates TRAF3. RIG-I and MDA5 activate TRAF3-TBK1 axis. Further, TRAF6 and TRAF3 induce the formation of NEMO-IKK $\alpha / \beta$ and NEMO-IKK $/$ /TBK1 complex, respectively. $I K K \alpha / \beta$ phosphorylates $I_{\kappa} B \alpha$ and releases active transcription factor, $N F-{ }_{\kappa} B . \mid K_{\varepsilon} /$ TBK1 phosphorylates IRF3. The activated transcription factors, $\mathrm{NF}^{-} \mathrm{KB}$ and IRF3 then translocate into the nucleus and induce proinflammatory cytokines and IFNs. PL2Pro, a viral product, inhibits TRAF6- and TRAF3mediated signal transduction, which leads to expression of inflammatory genes. TLR: tolllike receptor, $\mathrm{RIG}-1$ : retinoic acid-inducible gene 1, MDA5: melanoma differentialassociated protein 5, TRAF: tumor necrosis factor receptor-associated factor, NEMO: NF-kappa-B essential modulator, IKK: NF$\kappa \mathrm{B}$ inhibitor kinase, TBK1: TANK-binding kinase 1, NF- ${ }_{K} \mathrm{~B}$ : nuclear factor kappa-lightchain-enhancer of activated B cells, IRF-3: interferon regulatory transcription factor-3, TNF: tumor necrosis factor, IL: interleukin, IFN- $\beta$ : interferon beta. 
linkage has been shown to often target protein for degradation, while K63-linked Ub linkage has been found to mediate protein-protein interactions $[14,15]$.

Furthermore, ubiquitination of tumor necrosis factor receptor-associated factor 6 (TRAFG) and TRAF3 is required for activation of nuclear factor kappa-light-chain-enhancer of activated $\mathrm{B}$ cells $\left(\mathrm{NF}-{ }^{-} \mathrm{B}\right)$ and interferon regulatory transcription factor 3 (IRF3). When activated, TRAF6 auto-catalyzes its K63-linked ubiquitination. It has been reported to serve as scaffolds for $\mathrm{NF}^{-} \mathrm{\kappa} B$ essential modulator (NEMO)- I kappa B kinase (IKK) $\alpha / \mathrm{IKK} \beta$ complex binding. Moreover, K63-linked TRAF3 ubiquitination has been shown to create a scaffold for NEMO-mediated TANK-binding kinase 1 (TBK1)/IKK $\varepsilon$ complex recruitment [16]. The transcription factors IRF3 and NF-kB induced various cytokines including interferons (IFNs) and tumor necrosis factors (TNFs), which inhibit virus replication [17,18].

The potential targets of SARS-CoV PL2 ${ }^{\text {Pro }}$, which include IRF3, IкR $\alpha$, TRAF6, and TRAF3 have been reported (Fig. 4). SARS-CoV PL2 ${ }^{\text {Pro }}$ has been proposed to bind IRF3 and block its phosphorylation, dimerization, and nuclear translocation, and thereby inhibit IFN- $\beta$ induction [19]. Moreover, PL2Pro has been shown to inhibit NF- $\kappa \mathrm{B}$ signaling pathway by stabilizing its inhibitor, $\mathrm{I}_{\kappa} \mathrm{B} \alpha$ [20]. A protein comprising SARS-CoV PL2Pro and transmembrane region of Nsp3 has been demonstrated to interact with stimulator of interferon genes (STING)-TRAF3-TBK1 complex and remove the Ub from the ubiquitinated retinoic acid-inducible gene I (RIG-I), STING, TRAF3, TBK1, and IRF3 [21]. Furthermore, SARSCoV PL2Pro has also been shown to inhibit toll-like receptor 7 (TLR7)-mediated IFN response by removing K63-linked Ub chain from TRAF6 and TRAF3 [22].

\section{CONCLUSIONS}

As SARS-CoV-2 employs spike protein to bind and infect host cells, viral spike can be utilized as drug target. Since $\mathrm{PL}^{\text {Pro }}$ plays an important role in viral replication as well as inhibiting host innate immune response, it can be an efficient antiviral drug target for coronaviruses including SARSCoV, MERS-CoV, and SARS-CoV-2. Moreover, ubiquitination is an important inflammatory signal transduction. Thus, inhibition of ubiquitination and deubiquitination of Ublinked signal proteins such as TRAF6, TRAF3, IkB $\alpha$, and IRF-
3 can be a potential strategy for preventing inflammatory diseases. Possibly, the viral protein, PL2 ${ }^{\text {Pro }}$ may not only be a cause for severe symptoms in host, but also can be used to cure human diseases.

\section{CONFLICT OF INTEREST}

No potential conflict of interest relevant to this article was reported.

\section{REFERENCES}

1. Tang B, Bragazzi NL, Li Q, Tang S, Xiao Y, Wu J. An updated estimation of the risk of transmission of the novel coronavirus (2019-nCov). Infect Dis Model 2020;5:248-55.

2. Gallagher TM, Buchmeier MJ. Coronavirus spike proteins in viral entry and pathogenesis. Virology 2001;279:371-4.

3. Schoeman D, Fielding BC. Coronavirus envelope protein: current knowledge. Virol J 2019;16:69.

4. Gralinski LE, Menachery VD. Return of the coronavirus: 2019-nCoV. Viruses 2020;12:135.

5. Cyranoski D. Did pangolins spread the China coronavirus to people? [Internet]. Nature; 2020 [cited $2020 \mathrm{Feb} 7$ ]. Available from: https://doi.org/10.1038/d41586-020-00364-2.

6. Wang W, Tang J, Wei F. Updated understanding of the outbreak of 2019 novel coronavirus (2019-nCoV) in Wuhan, China. J Med Virol 2020;92:441-7.

7. Mousavizadeh L, Ghasemi S. Genotype and phenotype of COVID-19: Their roles in pathogenesis. J Microbiol Immunol Infect 2020. doi: 10.1016/j.jmii.2020.03.022. [Epub ahead of print]

8. van Boheemen S, de Graaf M, Lauber C, Bestebroer TM, Raj VS, Zaki AM, et al. Genomic characterization of a newly discovered coronavirus associated with acute respiratory distress syndrome in humans. mBio 2012;3:e00473-12.

9. Hoffmann M, Kleine-Weber H, Schroeder S, Krüger N, Herrler T, Erichsen S, et al. SARS-CoV-2 cell entry depends on ACE2 and TMPRSS2 and is blocked by a clinically proven protease inhibitor. Cell 2020;181:271-80.e8.

10. Guo YR, Cao QD, Hong ZS, Tan YY, Chen SD, Jin HJ, et al. The origin, transmission and clinical therapies on coronavirus disease 2019 (COVID-19) outbreak - an update on the status. Mil Med Res 2020;7:11.

11. Yuan W, Krug RM. Influenza B virus NS1 protein inhibits conjugation of the interferon (IFN)-induced ubiquitin-like ISG15 protein. EMBO J 2001;20:362-71.

12. Bakshi S, Holzer B, Bridgen A, McMullan G, Quinn DG, Baron $\mathrm{MD}$. Dugbe virus ovarian tumour domain interferes with ubiquitin/ISG15-regulated innate immune cell signalling. J 
Gen Virol 2013;94:298-307.

13. Yang X, Chen X, Bian G, Tu J, Xing Y, Wang Y, et al. Proteolytic processing, deubiquitinase and interferon antagonist activities of Middle East respiratory syndrome coronavirus papain-like protease. J Gen Virol 2014;95:614-26.

14. Komander D, Rape M. The ubiquitin code. Annu Rev Biochem 2012;81:203-29.

15. Yau R, Rape M. The increasing complexity of the ubiquitin code. Nat Cell Biol 2016;18:579-86.

16. Häcker H, Tseng PH, Karin M. Expanding TRAF function: TRAF3 as a tri-faced immune regulator. Nat Rev Immunol 2011;11:457-68.

17. Seth RB, Sun L, Chen ZJ. Antiviral innate immunity pathways. Cell Res 2006;16:141-7.

18. Hiscott J, Nguyen TL, Arguello M, Nakhaei P, Paz S. Manipulation of the nuclear factor-kappaB pathway and the innate immune response by viruses. Oncogene 2006;25:6844-67.
19. Devaraj SG, Wang N, Chen Z, Chen Z, Tseng M, Barretto N, et al. Regulation of IRF-3-dependent innate immunity by the papain-like protease domain of the severe acute respiratory syndrome coronavirus. J Biol Chem 2007;282:32208-21.

20. Frieman M, Ratia K, Johnston RE, Mesecar AD, Baric RS. Severe acute respiratory syndrome coronavirus papain-like protease ubiquitin-like domain and catalytic domain regulate antagonism of IRF3 and NF-kappaB signaling. J Virol 2009;83:6689-705.

21. Chen X, Yang X, Zheng Y, Yang Y, Xing Y, Chen Z. SARS coronavirus papain-like protease inhibits the type I interferon signaling pathway through interaction with the STINGTRAF3-TBK1 complex. Protein Cell 2014;5:369-81.

22. Li SW, Wang CY, Jou YJ, Huang SH, Hsiao LH, Wan L, et al. SARS coronavirus papain-like protease inhibits the TLR7 signaling pathway through removing Lys63-linked polyubiquitination of TRAF3 and TRAF6. Int J Mol Sci 2016;17:678. 